\title{
A MEG study of the Neural Substrates of Semantic Processing in Semantic Variant Primary Progressive Aphasia
}

\author{
Jessica Pineault ${ }^{1,2,3}$, Pierre Jolicoeur ${ }^{1,2}$, Stephan Grimault ${ }^{1,2}$, Jacinthe Lacombe ${ }^{1,2,3}$, \\ Simona Maria Brambati ${ }^{1,2,3}$, Nathalie Bier ${ }^{3,4}$, Céline Chayer ${ }^{4,5} \&$ Sven Joubert ${ }^{1,2,3}$ \\ ${ }^{1}$ Département de psychologie, Université de Montréal, Montréal, Canada \\ ${ }^{2}$ Centre de Recherche en Neuropsychologie et Cognition, Université de Montréal, Montréal, Canada \\ ${ }^{3}$ Centre de Recherche, Institut Universitaire de Gériatrie de Montréal, Université de Montréal, Montréal, Canada \\ ${ }^{4}$ Faculté de médecine, Université de Montréal, Montréal, Canada \\ ${ }^{5}$ Service de neurologie, Hôpital Maisonneuve-Rosemont (QC), Canada
}

RUNNING TITLE: Residual processing in svPPA 


\begin{abstract}
Despite a well-documented pattern of semantic memory (SM) impairment, the patterns of brain activation during semantic processing in svPPA still remain poorly understood. The current study aimed to investigate the neural substrates of residual semantic processing in the context of this significant but selective SM impairment, through the case study of one svPPA patient. One svPPA patient (EC) and six elderly controls carried out a general-level semantic categorization task (biological and manufactured objects) while their brain activity was recorded using magnetoencephalography (MEG). Despite similar behavioral performance, EC showed hyperactivation of the left inferior temporal gyrus (ITG) and right anterior temporal lobe (ATL) relative to controls. This suggests that periatrophic regions within the ATL region may support preserved semantic abilities in svPPA. These results thus contribute to our understanding of the brain regions which are recruited to compensate for bilateral atrophy of the ATL and ensure residual semantic processing in svPPA.
\end{abstract}

Keywords: semantic memory; single-case study; semantic variant primary progressive aphasia; functional neuroimaging. 


\section{$\underline{\text { Introduction }}$}

Semantic variant primary progressive aphasia (svPPA), also described in the literature as semantic dementia, is a neurodegenerative disorder characterized by an insidious breakdown of conceptual knowledge (Hodges, Patterson, Oxbury, \& Funnell, 1992; Snowden, Goulding, \& Neary, 1989; Warrington, 1975). The conceptual loss observed in svPPA is sensitive to specific properties of words, such as frequency/familiarity, prototypicality, and the level of specificity (taxonomy) of semantic processing required for these concepts (Ralph, Jefferies, Patterson, \& Rogers, 2017; Rogers et al., 2006). Regarding taxonomy, knowledge of properties that allow to individuate a specific concept from its semantic neighbors (e.g., a husky has blue eyes) is more vulnerable to decline than knowledge about properties shared by related concepts of the same category (e.g., a husky has four legs)(Catricalà et al., 2015). Therefore, patients are more impaired on tasks that require objects to be classified with greater precision (Patterson, Nestor, \& Rogers, 2007; Ralph and Patterson, 2008). This taxonomic impairment has been documented in an array of tasks recruiting various input/output modalities (naming, wordpicture matching, drawing, object recognition, coloring, lexical decision, and object use (Papagno and Capitani, 2001; Patterson et al., 2006; Rogers et al., 2004; Snowden, Neary, \& Mann, 1996; Warrington, 1975). It is also observed during spontaneous speech in svPPA patients, who tend to gradually replace the specific terms previously used to describe objects by more general words (e.g. thing), thus affecting their conversational intelligibility.

From a neuroanatomical standpoint, svPPA is marked by an extensive pattern of asymmetric bilateral gray and white matter degeneration, which typically begins (and is most prominent) on the lateral and ventral surfaces of the left anterior temporal lobe, but eventually progresses to include a larger portion of the temporal and inferior frontal cortices (Brambati et al., 2009; Desgranges et al., 2007; Diehl-Schmid, Onur, Kuhn, Gruppe, \& Drzezga, 2014; Hodges and Patterson, 2007; Whitwell and Josephs, 2012). More precisely, studies have shown, particularly in the early stage, a localized loss of 50 to $80 \%$ of gray matter volume on the inferolateral surface of the ATL (Hodges and Patterson, 
2007), as well as a number of alterations in the white matter tracts that connect anterior temporal regions to frontal (uncinate, arcuate fasciculi) and posterior temporal / occipital (inferior longitudinal fasciculi) regions (Acosta-Cabronero et al., 2011; Tu, Leyton, Hodges, Piguet, \& Hornberger, 2016). Furthermore, a direct relation between hypometabolism in anterior temporal regions (PET, i.e. functional damage) and atrophy of the same regions (MRI; i.e. structural damage) has been established (Diehl et al., 2004). More recently, alterations in the connectivity between regions of the semantic network have also started to emerge from concerted research efforts; studies suggest that, while neurodegeneration in svPPA originates in the left temporal pole, it appears to target preferentially the brain regions that are closer and more strongly connected to this region with disease progression (Collins et al., 2016). Therefore, behavioral deficits in svPPA could also result from this progressive “disconnection" pattern targeting the semantic network.

Due to the relatively circumscribed nature of semantic deficits in svPPA (at least in the early stages), and to the predominant underlying ATL atrophy, this syndrome has served as a critical pathological model of semantic memory (Patterson, et al., 2007; Ralph, et al., 2017). According to the most recent version of this model (Ralph, et al., 2017), the deployment of semantic knowledge in a variety of verbal and non-verbal everyday tasks relies on two interacting neural systems; the first is a representational one, while the second is associated with controlled/executive semantic processes. The representational system relies on the concerted interaction of a widely distributed neural network, in which sensory, motor, linguistic and affective sources of information (also referred to as the "spokes") are combined to form an integrated, heteromodal, representation. In accordance with the behavioral deficits and atrophy patterns documented in svPPA, the bilateral ventrolateral ATL region is considered to be the convergence site (a "hub") where the semantic information is processed at a conceptual level (Patterson, et al., 2007; Ralph, et al., 2017). This framework also postulates that semantic function varies in a graded manner across specific ATL subregions adjacent to the hub. For instance, because of its preferential connection to visual systems, the medial portion of ATL responds more to visual / 
concrete concepts, as opposed to STS/STG, which shares a greater connection to the language systems (Ralph, et al., 2017). The second control system was more recently introduced to the model, and is largely based on the study of patients with stroke aphasia (Jefferies and Lambon Ralph, 2006; Ralph, et al., 2017). This system manipulates activation within the representational system to generate inferences and behaviors that are tailored to each specific context (Ralph, et al., 2017). From an anatomical standpoint, it relies on regions such as the anterior cingulate-ventromedial prefrontal cortex, the posterior middle temporal gyrus (pMTG), the intraparietal sulcus and the pre-supplementary motor area, which are involved in strategic processes such as the manipulation and the controlled retrieval of semantic information from the semantic store (Jefferies and Lambon Ralph, 2006; Noonan, Jefferies, Visser, \& Ralph, 2013; Whitney, Kirk, O'sullivan, Lambon Ralph, \& Jefferies, 2010). Due to its widely distributed organization, the semantic network is thought to be relatively robust and resistant to neural damage. In addition, while unilateral ATL damage is associated with a slight decrease in performance (i.e. longer RTs, greater anomia, etc.), the intact contralateral "demi hub" appears to remain as operative as when the network was fully intact; it is therefore allowed to dominate performance, compensating for the decreased contribution of the damaged side. However, when both demi hubs are damaged, as is typically the case in later stages of the disease in svPPA, semantic processing is disrupted throughout, which results in much more significant impairment (Ralph, et al., 2017; Schapiro, McClelland, Welbourne, Rogers, \& Ralph, 2013). Although the "hub-and-spoke" model of semantic cognition is widely accepted, it is not the only existing perspective to explain semantic processing. While other models do not always agree on the existence of a single hub that supports the integration of modality-specific information into a multimodal representation (ATL), they generally conceptualize semantic memory as a network, which consists of both modality-specific and supramodal representations, and where semantic processing is supported by the gradual convergence of information across large regions of the temporal and inferior parietal association cortex (Binder and Desai, 2011; Binder, Desai, Graves, \& Conant, 2009) . 
To date, a number of studies have explored the semantic disturbances associated with structural alterations (gray matter loss) of the ATLs in svPPA patients (Adlam et al., 2006; Chan et al., 2009; Chen et al., 2017; Kumfor et al., 2016; Ralph, McClelland, Patterson, Galton, \& Hodges, 2001; Seeley et al., 2005). However, the functional correlates underlying semantic processing in svPPA remain poorly understood. In fact, very few studies have studied the dynamics of the brain regions underlying either impaired semantic processing or preserved (residual) semantic processing in svPPA (e.g. in the context of semantic tasks tapping superordinate properties of concepts). Among the few, Mummery et al. (1999) measured PET activation associated with a semantic decision task in svPA patients. Consistent with their impaired semantic performance, hypoactivation of the left ATL and posterior inferior temporal lobe was documented in svPPA patients relative to controls. However, relative to an equivalent visual task, they observed an increase in functional activation within the frontal regions and the bilateral ATL (more precisely, left anterior STG (BA21) and right TP (BA38)). Therefore, the results obtained from this study appear to provide some evidence that while semantic memory is indeed altered in svPPA patients, "residual" semantic processing abilities may elicit an additional recruitment of homologous and periatrophic temporal regions, as well as an increase in demands on the "controlled" semantic system (i.e. parietal/frontal regions). Other functional neuroimaging studies of svPPA patients focused on the semantic mechanisms associated with reading, in an attempt to identify the neuronal substrates underlying reading aptitudes in the context of normal and pathological aging (Wilson et al., 2008). In line with the behavioral manifestations of "surface dyslexia" observed in svPPA, results showed an increased activation of the left intraparietal sulcus, a region normally associated with subword mapping from orthographic to phonological representations, while patients were reading low-frequency/irregular words (Wilson, et al., 2008). This suggests that svPPA patients become increasingly dependent on subword orthographic-to-phonological processes (an alternative reading route) when knowledge related to the concept is impaired, suggesting compensation mechanisms at play. 
The aim of the current study was to conduct an in vivo investigation of the brain mechanisms associated with semantic processing in a case of svPPA, who presented with relatively circumscribed atrophy of the left ATL. More specifically, using a general-level semantic categorization task of manmade and biological entities requiring processing at a superordinate level, our aim was to gain a better understanding of the neural substrates underlying semantic processing in svPPA. We used magnetoencephalography (MEG), a non-invasive imaging technique used to record the magnetic fields generated by cortical electric activity. In contrast to other neuroimaging techniques such as fMRI, MEG has excellent temporal resolution. Another crucial advantage of MEG is that it allows a more accurate recording of the signal in the ATL region (i.e. the temporal poles), which is susceptible to significant signal reduction and has been found difficult to activate in classic fMRI protocols (Noppeney and Price, 2004; Visser, Jefferies, \& Ralph, 2010). The fMRI signal is distorted near airfilled cavities, such as the sinuses, due to discrepancies in the magnetic susceptibility across different tissue types (“susceptibility artifact”) (Devlin et al., 2000; Schmithorst, Dardzinski, \& Holland, 2001; Smith, Matthews, \& Jezzard, 2001)

To this end, the patterns of brain activation associated with a semantic judgment task involving the presentation of pictures of objects and animals were investigated in a svPPA patient and in a control group of healthy older adults matched for age and education. Prior to the presentation of each picture, the written name of a category (animal, tool, fruit/vegetable or means of transportation) was presented on the screen and each participant was instructed to correctly categorize the objects (e.g. the picture of a dog had to be matched with the written category "animal"). We focused on the signal around the N400, a component elicited by material requiring access to semantic memory and hence associated with semantic processing (Marinković, 2004). Initially described by Kutas and Hillyard (1984), the N400 has since then been extensively studied and is now conceptualized as a cortical response reflecting the activity of brain regions part of the semantic network following the presentation of a word or an image, regardless of modality (Kutas and Federmeier, 2000, 2011). The N400 is namely 
generated within the left ATL (Lau, Phillips, \& Poeppel, 2008; Luck, 2005) and thus appears to reflect a specifically semantic (heteromodal) process (Besson, Magne, \& Regnault, 2004; Kutas and Federmeier, 2011).

From a behavioral standpoint, our main hypothesis was that the svPPA patient would show relatively poorer performance than healthy controls in terms of accuracy and reaction times (RT) on the semantic task. We also predicted that MEG would reveal a disruption of the N400 component in the svPPA patient, associated with abnormal patterns of functional activation within key regions of the semantic network (including the ATL, IPC and the pMTG). Namely, given the ATL atrophy in svPPA, we hypothesized that other regions of the semantic network would respond to a greater extent in the svPPA patient during the task, reflecting a pattern of functional compensation and allowing for residual semantic processing to occur.

\section{Methods}

\section{Participants}

One svPPA patient and one group of six healthy elderly controls (HC) matched for age and education were recruited the current study. All subjects gave written informed consent before taking part to the protocol, which was approved by the Research Ethics Board of the Institut Universitaire de Gériatrie de Montréal (IUGM). Because semantic tests are particularly sensitive to cultural background, all participants included in the study had lived in Quebec for the previous 40 years (at least) and spoke French as their native tongue.

Patient. EC is a 67-year-old right-handed French-speaking woman, with 13 years of education. Retired at the time of testing, she was a former elementary school teacher. At the time of the study, she had already been an outpatient of the Memory Clinic of the IUGM in Montreal for two years. She was initially referred to the Clinic because she complained of word-finding difficulties, which had begun about two years earlier. The svPPA diagnosis was established by the neurologist (CC) based on current 
clinical criteria (Gorno-Tempini et al., 2011; Gorno-Tempini et al., 2004). EC was able to carry out independently many of her everyday activities. For instance, she drove and managed her budget alone, took part on a regular basis to "aquagym" lessons and was very much involved in her religious community. EC was quite introverted and rarely initiated conversation, but was described as an independent, proud and somewhat stubborn woman by her relatives, who confirmed that these personality traits were present prior to her svPPA diagnosis. EC was very aware of her deficits and verbalized concern about how they would impact her performance on some of the neuropsychological tasks (i.e., semantic). An exhaustive neuropsychological assessment was carried out over several sessions; EC's exhaustive neuropsychological assessment was reported in a previous publication, as she had later undergone a personalized semantic memory intervention (Bier et al., 2011). The extensive neuropsychological profiled is detailed in Table $\mathbf{1 .}$

Table 1. Neuropsychological test results for patient EC

\begin{tabular}{lcc} 
Neuropsychological testing & EC's results & Norms \\
\hline General functioning & 27 & Cut-off: 26/30 \\
MMSE & 17 & Cut-off: 24/30 \\
MoCA & $* 68$ & $\mathrm{z}>-2$ \\
\hline Global IQ (WAIS-III) & 91 & \\
$\quad$ Verbal IQ & $* 92$ & $\mathrm{z}=0.80$ \\
$\quad$ Performance IQ & & $\mathrm{z}>-2$ \\
\hline Visuospatial functions & 36 & \\
Perceptual Organization Index (WAIS-III) & $* 22$ & \\
Rey-Osterrieth Complex Figure (ROCF) & $* 15$ & \\
$\quad$ Copy (36) & & \\
Birmingham Object Recognition Battery (BORB): Object decision subtest & \\
OD A Easy (32) & & \\
OD A Hard (32) & &
\end{tabular}


OD B Easy (32)

\section{Episodic memory}

Delayed recall of ROCF (36)

$z=-0.26$

\section{Working memory}

Working Memory Index (WAIS-III)

78

Attentional and executive functions

Processing Speed Index (WAIS-III)

DKEFS Color-Word Interference (sec.)

Naming (colors)

Reading

Inhibition

Inhibition/Switching

Errors (corrected/uncorrected)

D2 Test: concentration endurance test

Total raw score

Errors

Trail Making Test (sec.)

Part A

50

101

Part B

Language

Letter fluency

Category fluency (total)

Repetition of words (15)

Repetition of non-words (10)

Delayed repetition of words (15)

Delayed repetition of words (10)

Reading

Regular words (5)
*16

$* 4$

15

10

15

10 ss $=8$

$\mathrm{Ss}=11$

$\mathrm{ss}=5$

ss $=10$

ss $=8$ $z=-1.24$

$z=-0.44$

$z=0.86$

$z=-0.10$ $z>-2$

$z>-2$

$$
\mathrm{z}=0
$$$$
\mathrm{z}=0.5
$$$$
\mathrm{Z}=0
$$$$
\mathrm{z}=0.7
$$ 
Irregular words (5)

Nonwords (10)

Writing to dictation

Regular words (10)

Irregular words (10)

Nonwords (10)

DO-80 picture naming test (80)

Pyramid and Palm Trees Test (52)

Picture-picture

Word-word

Verbal Comprehension Index (WAIS-III)

\section{Affect}

Geriatric depression scale
*3

8

9

*9

9

*16

*34

$* 28$

*69 $\mathrm{z}>-2$

$\mathrm{z}=-1.3$

$z>-2$

$z>-2$

$z=-0.6$

$\mathrm{z}>-2$

$z>-2$

$z>-2$

$z>-2$

Self-Rated (30)

4

Cut-off: 15
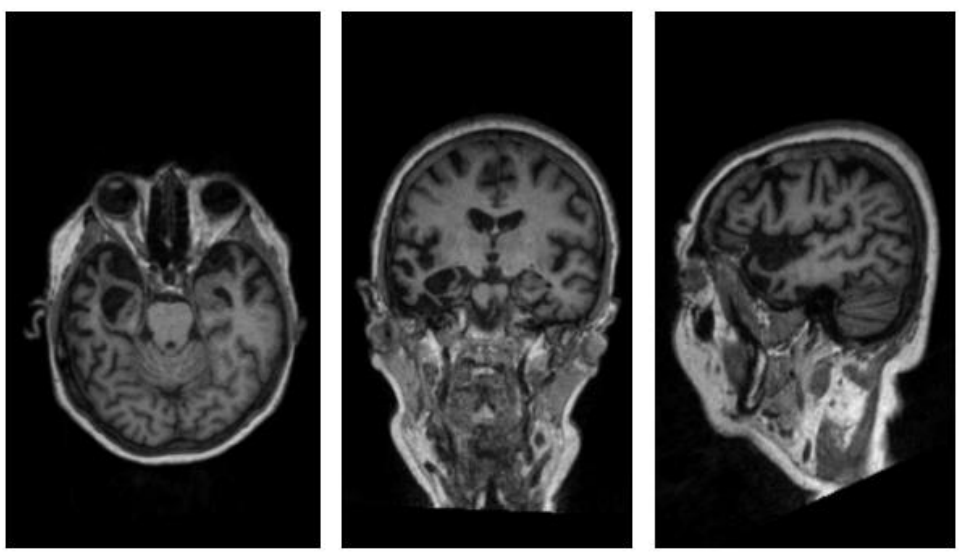

Patient EC

Figure 1. Structural MRI image of EC's brain, exhibiting a pattern of bilateral but asymmetric (left-predominating) atrophy of the ATLs, consistent with the pattern of atrophy classically observed in svPPA (Gorno-Tempini, et al., 2011; Hodges, et al., 1992). 
Healthy elderly controls. The control group included 6 healthy elderly individuals (1M, 5F; mean age $=69.33, \mathrm{SD}=3.78)$, who had completed an average of 14.83 years of education $(\mathrm{SD}=3.71)$. They were matched to patient EC for age and education level, and with normal cognitive function, as verified through a standard neuropsychological assessment. Controls were recruited via the CRIUGM participant database and through written public announcements. A Mann-Whitney U test indicated that the control group did not differ significantly from $\mathrm{EC}$ in terms of age $(\mathrm{U}=5.5, \mathrm{z}=1.038, \mathrm{p}=.571)$ or education $(\mathrm{U}=3, \mathrm{z}=0, \mathrm{p}=1)$. Basic exclusionary criteria for both $\mathrm{EC}$ and the control group included a history of systemic or neurological disease, past or current psychiatric illness, traumatic brain injury, history of alcoholism, untreated medical or metabolic condition, general anesthesia in the last year, as well as uncorrected hearing and/or vision problems.

\section{Semantic decision task}

This computerized task was developed in the Laboratory and specifically adapted to MEG. It was devised to evaluate the integrity of semantic processing through a semantic categorization task addressing knowledge of everyday objects. Stimuli were back projected on a computer screen located at eye level at a distance of $60 \mathrm{~cm}$ from the subject in the MEG using the E-Prime software (Psychology Software Tools, Pittsburg, PA). Stimuli were presented one by one. Stimuli consisted of 192 high resolution colored photographs of everyday objects belonging to four semantic categories: animals, transportation, fruits/vegetables and tools. All pictures were equivalent in terms of resolution (number of pixels) and background luminosity and contained no contextual clue which may have helped participants in their decisions (i.e. no background information).

The semantic decision task was administered to each subject individually during a two-hour meeting in the MEG lab located in the Université de Montréal Psychology Department. Upon arrival, participants were tested for MEG compatibility before they were given the instructions relative to the computerized task. Once installed into the magnetically shielded room, each participant completed a 
testing session consisting of 48 practice trials followed by 192 experimental trials. Each trial started with concise written instructions reminding the participants to keep their eyes fixated on the cross located at the center of the screen and to blink only once feedback was given to them. Participants initiated trials by pressing simultaneously with their thumbs on both side buttons on the side of the response case. At the beginning of each of the four blocks, the written name of a semantic category (e.g., 'animal') was presented on the screen during 1000ms. Participants were instructed to decide if each of the 48 stimuli presented subsequently for a duration of $500 \mathrm{~ms}$ belonged to that semantic category and to provide an answer by pressing the appropriate key on the response cases. Each of the 96 images (24 per category) was presented twice in the experiment: once in a congruent condition (e.g., animal-tiger) and once in an incongruent condition (e.g., tool - tiger). Participants were given 2500ms to respond and were instructed to respond as accurately yet as quickly as possible after each stimulus presentation. The order of blocks and the hand used to for each type of answer (matching, nonmatching) was counterbalanced between subjects, as well as the order of presentation of the stimuli in each 48-trial block, which was randomized for each subject. To minimize the impact of performance anxiety and ensure that participants remained focused throughout the task, feedback relative to accuracy was not given. The only feedback shown to them was a blank circle post-response, informing them that their answer had been correctly recorded (refer to Figure $\mathbf{2}$ for a schematic representation of a trial design). 


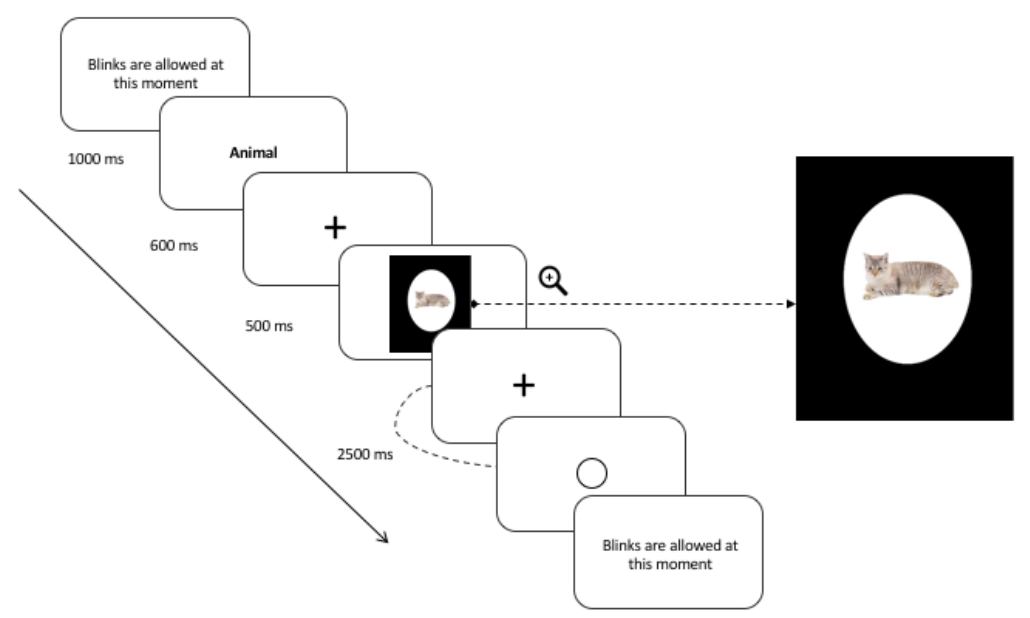

Figure 2. Trial design illustrating an example of a trial for the congruent condition.

\section{Data Acquisition}

MEG data. The participants' functional brain activity was recorded while they completed the semantic decision task using a whole-head CTF-VSM 275 sensors MEG system in a magnetically shielded room. MEG signals were acquired continuously during the task at a sampling rate of $1200 \mathrm{~Hz}$. Prior to recording, subjects were screened for MEG artifacts (dental work/metal implants). When necessary, plastic lenses were installed on the subjects' eyes to avoid magnetic perturbations from the metal parts of their personal glasses and ensure their optimal visual processing of the stimuli during the task. Bipolar electroocculogram (EOG) (electrodes placed at the left and right canthi for horizontal EOG and above and below the left eye for vertical EOG) as well as unipolar ECG were also recorded.

Structural MRI. Following the MEG protocol, each control and aMCI participant underwent a high resolution anatomical MRI using an optimized MPRAGE protocol with a 3.0 T Siemens TRIO MRI (Siemens, Erlanger, Germany) located at the Unité de neuroimagerie fonctionnelle in the Research Centre of the IUGM (http://www.unf-montreal.ca/siteweb/Home_en.html). A volumetric magnetization 
prepared rapid gradient echo (MP-RAGE) sequence was used to acquire high-resolution T1-weighted $3 \mathrm{D}$ anatomical images, using the following parameters: $\mathrm{TR}=2.3 \mathrm{~s}, \mathrm{TE}=2.94 \mathrm{~ms}$, TI=900ms, flip angle $=9^{\circ}, \mathrm{FOV}=240 \times 256$, voxel size $=1 \times 1 \times 1.2 \mathrm{~mm} 3$. An 8 -channel head coil was used. The scans were then used to constrain source localization analyses of the MEG data. Using their respective MRI images, a 3D representation of each subject's cortex was realized through mathematical morphometry (refer to the Source localization section for more details).

\section{Data Analyses}

Behavioral Data. Response times and number of correct responses were averaged across each participant to allow comparison with patient EC. Items left unanswered were recorded as incorrect responses. Mann-Whitney U tests were performed on both accuracy and response times.

Voxel-based morphometry. VBM was applied to detect regional gray matter (GM) atrophy in the patients compared to 15 age-matched healthy controls. The VBM group was composed of neurotypical older female participants (mean age $=71.1, \mathrm{SD}=2.6$ ) without neurological diseases or cognitive impairment (mean MoCA score $=27.4 ; \mathrm{SD}=1.8$ ). VBM analyses were conducted using SPM12 software package (Wellcome Department of Imaging Neuroscience, London; http://www.fil.ion.ucl.ac.uk/spm). The images were segmented into gray (GM) and white (WM) matter. Affine registered tissue segments were used to create a custom template using the DARTEL (diffeomorphic anatomical registration using exponentiated lie algebra) approach, including the image of the patient and of the control group (Ashburner, 2007). For each participant, the flow fields calculated during the DARTEL template creation step were applied to each participant's GM image. The VBM analysis was based on modulated GM images, where the GM value for each voxel was multiplied by the Jacobian determinant derived from spatial normalization to preserve the total amount of GM from the original images (Ashburner and Friston, 2000). The resulting modulated and normalized images were then smoothed with a Gaussian kernel of $8 \mathrm{~mm}$ FWHM. A t-test was used to 
compare the pre-processed image of the patient to the control group, in order to obtain and visualize the pattern of GM atrophy of the patient. A significance threshold of $p<0.001$ uncorrected was applied.

MEG Data. MEG analyses were conducted using CTF tools, BrainVisa (http://brainvisa.info) and AFNI software (Cox, 1996). Third-order gradient noise reduction (computed with CTF software) and low-pass filter at $20 \mathrm{~Hz}$ was applied to all MEG signals before segmentation. Subsequently, signals were segmented according to the chosen time window (-200 to $1000 \mathrm{~ms}$ relative to the onset of the stimulus) with the $200 \mathrm{~ms}$ preceding stimulus onset used as a baseline period. Trials with eye movement were systematically removed following a careful inspection of the vertical and horizontal EOG traces. Trials with large movement artifacts, such as head motion exceeding $5 \mathrm{~mm}$ from baseline recording and other external noise were removed as well. Only trials with a correct response were included in the following brain signal analyses.

Because the task was aimed to assess EC's ability to adequately categorize objects, a specific time window reflecting this type of semantic processes was selected based on visual inspection of maps displaying the averaged MEG signal, as well as supporting evidence in the literature. This time window, extending from $495 \mathrm{~ms}$ to $695 \mathrm{~ms}$, was determined as the probable latency of the $\mathrm{N} 400$ component, which is known to reflect the semantic processing of meaningful semantic stimuli (Kutas and Federmeier, 2011; Kutas and Hillyard, 1984; Lau, et al., 2008). Previous ERP studies documenting the particularities associated with semantic processing in aging have demonstrated that the peak latency of this component is generally delayed, supporting our choice of a slightly delayed and extended time window range (Federmeier and Kutas, 2005; Federmeier, Van Petten, Schwartz, \& Kutas, 2003; Giaquinto, Ranghi, \& Butler, 2007; Harbin, Marsh, \& Harvey, 1984; Kutas and Iragui, 1998; Taler, Klepousniotou, \& Phillips, 2009). Subsequently, MEG signals were averaged over time for this preselected time window (495 to 695ms), for every participant and each trial. 
Source localization. Source localization analyses were performed on these averaged MEG signals using the maximum entropy on the mean method (MEM) (Amblard, Lapalme, \& Lina, 2004; Grova et al., 2006). This method is a cortically constrained distributed source-localization approach. The cortical surface was segmented from each anatomical MRI scan using BrainVisa software (http://brainvisa.info). Approximately 4000 sources were distributed over the entire cortical surface, and these local sources were used in distributed source localization analyses, which were conducted separately for each participant and every trial. Following the analyses of the localizations performed on a participant-wise basis, the following procedure was used to perform a group analysis of the localization. Each cortical-surface localization map was interpolated in the volume MRI image for each subject and the resulting image was smoothed using a Gaussian filter with an $8 \mathrm{~mm}$ FWHM (full width at half maximum). Finally, all the images were normalized to a common template in Talairach space (ICBM 152, Montreal Neurological Institute) using SPM2.

Statistical analyses. Covariation analyses were performed using a General Linear Model (AFNI) considering the normalized source-localization maps. Because of limitations in the AFNI software in terms of the GLM size (the number of lines considered in the analysis), a subset of 110 trials per subject was included in the GLM (fifty-five trials per condition for each participant, for a total of 770 entries). Comparisons between EC and the control group were performed using a voxel-wise threshold at $\mathrm{p} \leq .05$, corrected for multiple comparisons with false discovery rate (FDR) method (Benjamini and Hochberg, 1995). Clusters of voxels with a volume of at least $632 \mathrm{~mm}^{3}(\mathrm{t}=2.607)$ were considered as statistically significant based on these criteria.

\section{$\underline{\text { Results }}$}

Semantic decision task. Both EC (accuracy score $=95 \%, M_{R T}=1003.43 \mathrm{~ms}$ ) and the healthy controls ( $\left.M_{\text {accuracy score }}=94.83 \%, M_{R T}=891.71 \mathrm{~ms}\right)$ performed exceptionally well on the semantic task. Thus, 
Mann-Whitney $U$ tests conducted on the behavioral data collected during the task showed no significant differences in terms of total accuracy scores $(\mathrm{U}=2.5, \mathrm{z}=-.255, \mathrm{p}=.857)$ or mean response time $(\mathrm{U}=5.0, \mathrm{z}=-.1, \mathrm{p}=.571)$.

Voxel based morphometry. Patient EC showed a pattern of significant gray matter atrophy extending throughout the left ATL. More precisely, atrophy encompassed the anterior portion of the superior, middle and inferior temporal gyrus, as well as the temporal pole and the left hippocampal region (refer to Table 2 and Figure 3 for an exhaustive representation of the atrophied structures).

Table 2. Regions of significant atrophy for patient $\mathrm{EC}^{1}$.

\begin{tabular}{lcccc}
\hline Region (Brodmann Areas) & $\mathbf{x}$ & $\mathbf{y}$ & $\mathbf{Z}$ & Z-score \\
\hline L. Superior Temporal Gyrus (BA22) & -46 & 3 & -2 & 4.1 \\
& -36 & 12 & -29 & 3.6 \\
\hline L. Middle Temporal Gyrus (BA21) & -27 & 17 & -36 & 3.6 \\
& -51 & -21 & 1 & 3.6 \\
\hline L. Inferior Temporal Gyrus (B20) & -51 & -30 & -20 & 3.3 \\
& -46 & -7 & -41 & 3.3 \\
\hline L. Temporal Pole (BA38) & -27 & 21 & -39 & 3.6 \\
& -48 & 18 & -14 & 3.5 \\
\hline L. Hippocampus / Amygdala & -27 & 2 & -21 & 3.1 \\
\hline
\end{tabular}

\footnotetext{
${ }^{1}$ A significance statistical threshold of $\mathrm{p}>.001$ uncorrected was used for the VBM analyses.
} 


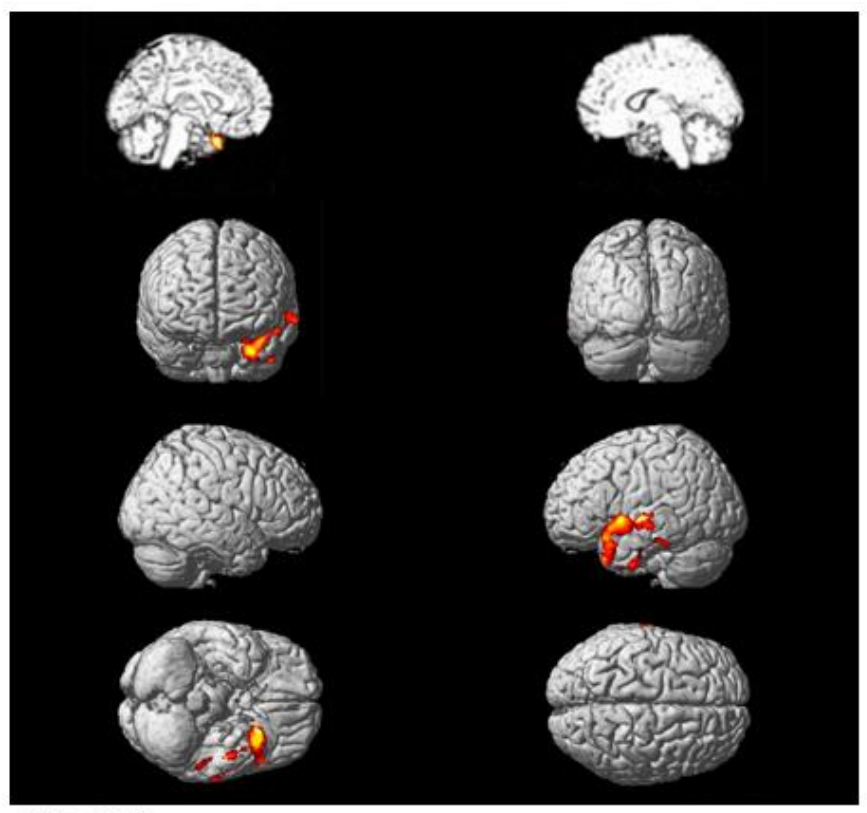

Patient EC

Figure 3. Whole-brain VBM analysis showing regions of significant atrophy for patient EC compared with healthy controls.

Functional (MEG) activation. Direct contrasts were carried out between EC and the control group on a time window stretching from 495-695ms (see Methods for more details). Maps of mean functional activation for both groups are displayed in Figure 4a. Despite performing equally well in the MEG task, as expressed by accuracy scores, a pattern of bilateral temporal lobe hyperactivation was found in EC relative to the control group. In contrast, no significantly greater pattern of hyperactivation was found in the control group relative to EC. More specifically, EC activated the left ITG as well as the right ITG/AT to a greater extent than the control group. Localization maps depicting those differences are shown in Figure $\mathbf{4 b}$, while peaks of hyperactivation are listed in Table $\mathbf{3}$. The Talairach coordinates and Brodmann area labels were provided by AFNI software (Cox, 1996). Also, although not statistically significant, a difference approaching significance ( $q \leq .11$ FDR) was found between conditions (incongruent>congruent) in the right ATL region. However, additional analyses revealed a 
pattern of group x condition interaction. More precisely, in both groups, subtracting the cortical activity generated in the congruent condition from the cortical activity generated in the incongruent condition resulted in a typical "N400 effect" pattern (incongruent>congruent) within the right ATL; this N400 effect was, however, intensified (incongruent $>>$ congruent) for patient EC. Within the left ATL region, while a typical N400 effect pattern was observed for the control group (incongruent $>$ congruent), the opposite pattern was observed for patient EC (whereas processing of congruent stimuli with the context appeared to generate greater activation than incongruent ones; congruent $>>$ incongruent). Localization maps depicting those differences are shown in Figure 4c.

Table 3. Differences of activation between EC and the control group ${ }^{2}$

\begin{tabular}{|c|c|c|c|c|c|}
\hline Region (Brodmann Areas) & $\mathbf{x}$ & $\mathbf{y}$ & $\mathbf{Z}$ & Cluster Size & T value \\
\hline \multicolumn{6}{|l|}{ EC $>$ Control group (congruent + incongruent context) } \\
\hline R. Inferior Temporal Gyrus / Temporal Pole (BA20/38) & 39 & -15 & -43 & 140 & -15.78 \\
\hline L. Inferior Temporal Gyrus (BA20) & -45 & -3 & 37 & 130 & -14.53 \\
\hline \multicolumn{6}{|l|}{ Interaction effect (group $x$ condition) ${ }^{3}$} \\
\hline R. Superior Temporal Gyrus (BA38) & 35 & 6 & -46 & 81 & 5.73 \\
\hline L. Inferior Temporal Gyrus (BA20) & -45 & -7 & 37 & 42 & -1.98 \\
\hline
\end{tabular}

${ }^{2}$ Peaks of hyperactivation in EC relative to the control group. A significance statistical threshold at $\mathrm{q} \leq .05$ FDR has been used. Talairach (MNI) coordinates are provided.

${ }^{3}$ Peaks of hyperactivation for the crossover interaction. A significance statistical threshold at $\mathrm{q} \leq .05$ FDR has been used. 

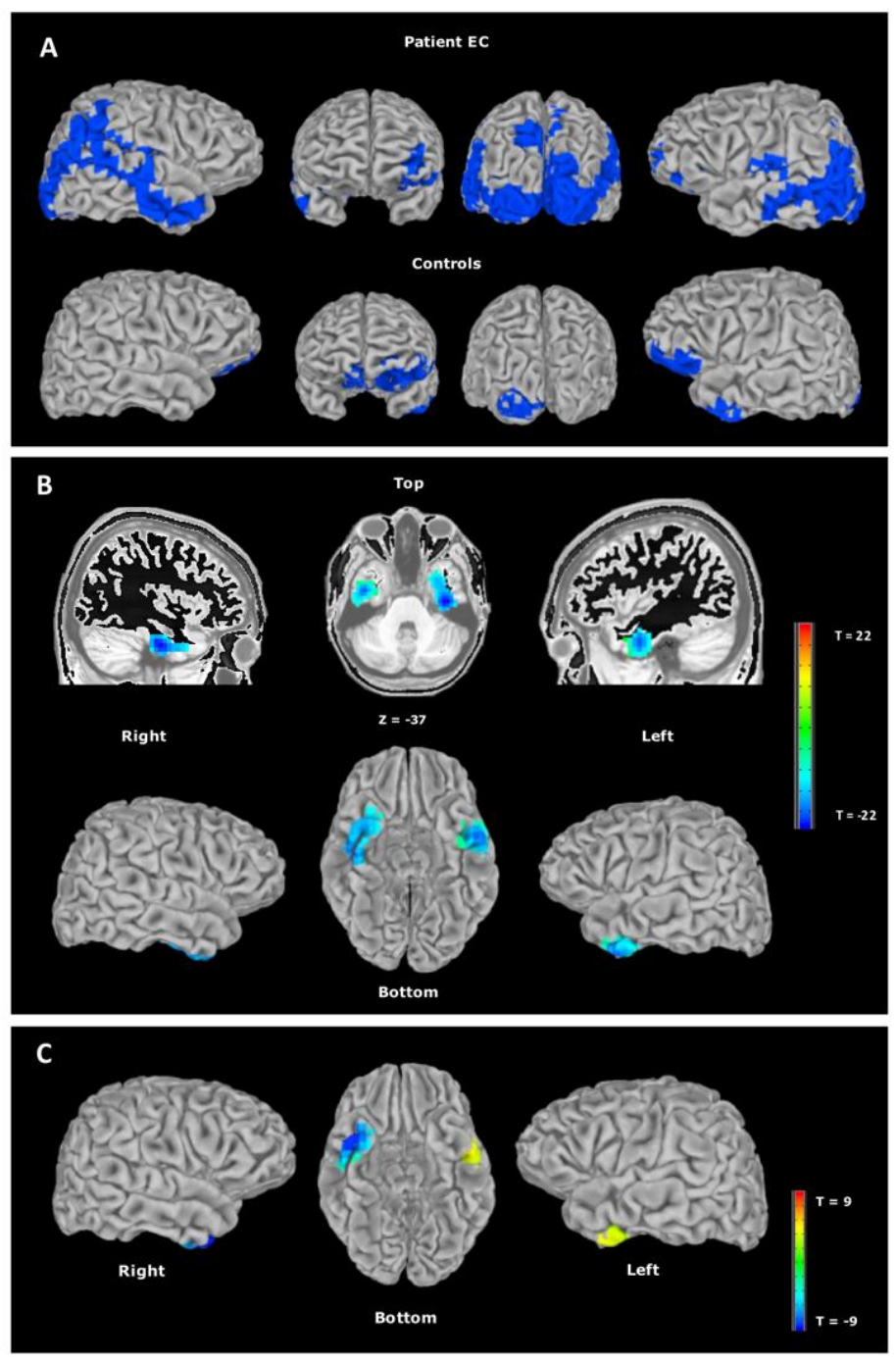

Figure 4a. Patterns of mean functional activation for EC and the Control group related to the semantic categorization task. For all three images, results are depicted for the 495-695ms time window. 4b. Differences in MEG activation between EC and the Control group. Areas in blue indicate significantly greater activation in patient EC relative to the Control group (left $=$ left hemisphere). In contrast, there were no areas showing a significant pattern of hyperactivation in the Control group relative to patient EC. 4c. Interaction effect (group $x$ condition). The blue area indicates that a typical "N400 effect" (activation: incongruent>congruent) was found for each group; but this effect was amplified in patient EC. Areas in yellow indicate that, while a typical N400 effect was observed within the left ATL for the control group, a reversed (activation: congruent>incongruent), amplified pattern was observed for patient EC. 


\section{$\underline{\text { Discussion }}$}

In the current study, we used MEG to conduct an in vivo investigation of the brain mechanisms associated with semantic processing in a case of svPPA. To this aim, one svPPA patient (EC) and a control group of healthy seniors strictly matched for age and education completed a computerized semantic categorization task while their neural activity was recorded with the MEG system. It is important to know that the task was designed in a way that required EC to adequately categorize, but not to name, describe or share explicit knowledge concerning each object. Surprisingly, the performance of patient EC and of the control group was strictly identical at the behavioral level. At the brain level, however, EC activated the left and right ATL region to a greater extent than the control group. At the behavioral level, these results may seem surprising at first glance, considering the magnitude of EC's impairment on neuropsychological measures of semantic memory (see Table 1). However, even though semantic impairment in svPPA is found across a wide range of neuropsychological tasks, overall performance of patients has been shown to depend on the level of specificity required by the semantic task (Rogers, et al., 2006; Rogers and Patterson, 2007; Rogers, Patterson, Jefferies, \& Ralph, 2015). For instance, svPPA patients are known to perform fairly well on tasks requiring a relatively coarse, domain-level categorization of the stimulus (for instance, knowing that a Chihuahua is an animal but that a carrot isn't), as opposed to a more precise classification (knowing that a Chihuahua is a dog and not a cat) or subordinate-level classification (knowing that a Chihuahua is a Chihuahua but that a Yorkshire isn't). This can be explained by the fact that discriminating concepts with a higher degree of overlap (i.e. concepts within the same basic- or subordinate-level category) is known to exert increased demands on identification and differentiation processes at the level of both semantic and perceptual processing (Ikeda, Patterson, Graham, Ralph, \& Hodges, 2006; Tyler and Moss, 2001; Tyler et al., 2004), as opposed to concepts with little overlap (for instance, items belonging to different domains) which lead to different responses, and for which even 
an imprecise/weakly-specified or partial conceptual representation can translate into an appropriate response or behavior (Hodges, et al., 1992; Pobric, Jefferies, \& Ralph, 2007; Rogers, et al., 2006). Accordingly, recent studies have demonstrated that the level of specificity required by the task may influence the degree to which the ATLs are activated. In this regard, although the ATLs are preferentially recruited when concepts are processed at a specific (subordinate) level (Grabowski et al., 2001; Patterson, et al., 2007), they remain involved to a lesser extent during processing of concepts at the domain (superordinate) level (Pobric, et al., 2007; Ralph, 2014; Rogers, et al., 2006; Visser and Ralph, 2011). For instance, Rogers and Patterson (2007) showed that, while healthy controls are faster and more accurate at categorizing items at the basic level (for example, $d o g$ ) relative to a more general (for example, animal) or specific level (for example, Chihuahua), patients with svPPA show the opposite pattern and are more impaired when basic-level classification is required by the task. Such findings suggest that semantic tasks that require a distinctive classification of closely related stimuli (semantic neighbors) place particularly strong demands on the ATL region, which is affected in svPPA. Because of the ATL implication in generalizing/differentiating between concepts, it becomes difficult for patients, as the disease progresses, to differentiate between similar/neighboring concepts on the basis of idiosyncratic details, since the damaged semantic system is no longer able to generate sufficiently precise conceptual representations to support these processes. Nevertheless, the degraded system is still able to generate an adequate response when it comes to more general/common characteristics, because their high level of resemblance within a conceptual category makes them more readily accessible and less vulnerable to damage (Marques and Charnallet, 2013; Murre, Graham, \& Hodges, 2001; Rogers, et al., 2015). In the case of the semantic judgment task used in the current study, there was little overlap across the different categories (e.g. Animals vs. Tools), therefore stimuli could be processed at a very basic, superordinate level and still yield the right answer. Thus, patient EC could have relied on coarse knowledge to accomplish the task, or on a general criterion (e.g. edible = fruit/vegetable or has eyes = animal) to guide her decisions for each category, which may explain why 
she performed so well on the task despite showing significant atrophy of the ATL region.

The main goal of this study was to investigate the neural correlates associated with semantic processing in svPPA using MEG, a neuroimaging technique offering both excellent temporal and spatial resolution. Analyses of the patterns of functional neural activity associated with semantic processing revealed differences between EC and the control group. Firstly, patient EC activated the bilateral ATL region to a greater extent than the control group. Secondly, no brain regions showed significant hypoactivation in EC relative to the control group. Finally, the control group did not activate any region to a greater extent than EC (see maps of activation for the control group in Figure 4b). EC's pattern of functional hyperactivation suggests that semantic processing in svPPA exert demands on peri-atrophic and homologous ATL regions. These results are similar to those found by Mummery et al. (1999). Despite these similarities, the svPPA patients in their study also showed hypoactivation of the left ITG and right temporo-parietal junction, which was not the case in the current study. In addition, the patients' performance was significantly lower than that of the control group, leading the authors to conclude that the additional recruitment of right temporal structures proved insufficient to counterweight ongoing neurodegenerative processes and allow svPPA patients to process semantic information as effectively as healthy seniors. In addition, the authors also observed an increase in activation of prefrontal regions (BA44/45/47/6) in their svPPA patients compared to controls, which was not replicated in the current study. The involvement of prefrontal regions in semantic control is fairly well established (Binder, et al., 2009; Noonan, et al., 2013; Ralph, et al., 2017). This pattern of prefrontal cortex (PFC) hyperactivation was interpreted by the authors as a reflecting task difficulty and increased demands on the executive mechanisms of selection, discrimination and strategic control underlying the retrieval of information in long-term memory (Mummery et al., 2000). Such mechanisms are considered necessary to select the best answer between very similar concepts (within the same basic-level category). Within the context of the present study, it is possible that the relatively 
simple degree of semantic processing to carry out the task did not exert significant demands on the PFC-based semantic controlled mechanisms.

Furthermore, the pattern of functional hyperactivation found in patient EC involving periatrophic regions in the left ATL as well as additional contralateral activation in the more preserved right ATL may reflect a mechanism of functional compensation, by which EC is able to achieve a similar level of performance as healthy elderly individuals. This suggests that despite significant structural loss in this key region of the semantic network, residual abilities underlying successful coarse/domain-level semantic processing in this patient may be underpinned by the additional recruitment of peri-atrophic regions and homologous regions within the ATL. Although operational criteria to define compensation in the context of pathological aging are still debated, compensation in neurodegenerative disease is increasingly recognized as taking place when patients' behavioral performance remains similar to that of the normal population in the presence of structural degradation (Barulli and Stern, 2013; Gregory et al., 2017; Scheller, Minkova, Leitner, \& Klöppel, 2014). In the current case study, it is possible that compensatory processes were at play in this svPA patient because atrophy was strongly left-lateralized and the right ATL region sustained minimal damage (see Table 2 and Figure 3). There is indeed increasing evidence highlighting the importance of bilateral ATL involvement in semantic processing (Bi et al., 2011; Ralph, et al., 2017; Rice, Lambon Ralph, \& Hoffman, 2015; Schapiro, et al., 2013). In addition, studies have documented the mechanisms by which additional recruitment of the less damaged contralateral demi hub allows to help compensate (at least in part) for the decreased contribution of the damaged hub in the context of neural insult (Binney and Ralph, 2015; Schapiro, et al., 2013). For instance, in a recent TMS study conducted on healthy participants, Binney and Ralph (2015) used rTMS to suppress left ATL activity, while recording neural activity (using fMRI) as they completed a semantic judgment task. Their results shed some light on the dynamics of the unperturbed semantic system, by demonstrating that while task performance was initially underpinned by the bilateral ATL region, network activity became essentially unilateral with 
time (left-lateralized in their case). This intrinsically-modulated change in the distribution of neural activity was interpreted as "an initial generous allocation of processing resources preparing the system for the most challenging of (initially unknown) task demands, followed by a gradual decrease until the most parsimonious and efficient state of activity is reached" (Binney and Ralph, 2015) p.179). However, following TMS-induced suppression of the left ATL activity, an active maintenance of activation was observed within the right ATL whereas, under normal circumstances, activity was observed to diminish over time. Therefore, it is possible that the increased activation of the right ATL regions in EC (relative to controls) may reflect a similar process of "up-regulation" of the contralateral regions following a predominantly left-lateralized breach of the semantic network (Binney and Ralph, 2015).

As evidenced by a pattern of group $x$ condition interaction (see Figure 4c), it is possible that the pattern of hyperactivation found may reflect different mechanisms with the left and right ATL in patient EC. The pattern of activation in the right ATL suggests a typical N400 effect, whereby the integration of incongruent (relative to congruent) information with context (semantic category) is associated with an increase in neural activity. Although amplified in EC relative to the control group, the activity found in this region is consistent with what has been reported concerning the role and distribution of the N400 effect in the literature (see Kutas and Federmeier (2011) for a review). While a typical N400 effect was observed for the control group in the left ATL, a reversed and amplified N400 pattern was found for patient EC (i.e. congruent condition resulted in greater activation than incongruent condition). In line with the functional compensation hypothesis, it is possible that semantic integration requires the deployment of additional neural resources within the semantic network when there is an incongruity between a stimulus and its context. In the patient, this operation would require greater support/involvement from the right ATL (up-regulation) than in a semantically congruent context. In the latter case, it is possible that the left periatrophic ATL regions may be sufficient to ensure successful semantic performance. Nonetheless, the nature of the possible collaboration between 
periatrophic and contralateral regions within the left and right ATL, as well as their specific compensatory roles, will need to be further investigated in future studies in order to better understand the specific mechanisms at play.

Finally, the current study has some limitations which deserve to be mentioned. First of all, as is the rule with all single case studies, the results presented need to be interpreted carefully and should be replicated in a larger sample of svPPA patients in order to confirm the specific brain mechanisms described here. Longitudinal studies of svPPA patients may also bring valuable insights into our understanding of the evolution of compensatory mechanisms as the disease progresses. Finally, although beyond the scope of the current study, future studies could also investigate how varying levels of difficulty in the semantic tasks may impact resulting patterns of functional hyperactivation and compensation in the context of neurodegeneration. This may provide further insights regarding the functional mechanisms underlying the semantic network in pathological and normal aging.

\section{Conclusion}

In summary, we investigated the neural correlates of semantic processing in a case of svPPA with predominantly left-lateralized atrophy and in a group of age and education-matched healthy older controls. Despite undistinguishable performance at the behavioral level (accuracy rates, RT), functional neuroimaging (MEG) analyses showed a pattern of significant hyperactivation within the left and right ATL region in the svPPA patient relative to the control group. No such patterns of hyperactivation were found within other regions of the semantic network, including semantic control regions (PFC, pMTG). These results suggest that compensatory mechanisms within specific regions of the semantic network are at play in svPPA, allowing to successfully perform basic-level semantic tasks, despite damage to more fine-grained semantic processing. Results of the current study are also consistent with the idea of a concerted and balancing role of the left and right ATL in semantic processing. Accordingly, the contralateral and perilesional portions of the ATL region may help compensate for 
decreased involvement of the damaged hub. Therefore, taken together, these results suggest that regions of the semantic network adjacent to atrophic regions may help compensate neurodegeneration in svPPA when some aspects of semantic processing are still possible.

\section{Acknowledgments and funding}

SJ and PJ were supported by the Alzheimer Society of Canada. SJ was supported by a Chercheur boursier senior award from the Fonds de Recherche du Québec en Santé (FRQ-S) and JP was supported by a Canadian Institute of Health Research (CIHR) doctoral award.

\section{Disclosure}

The authors declare they have no actual or potential conflicts of interest that could prejudice or bias the results and outcomes reported in this manuscript. 


\section{References}

Acosta-Cabronero, J., Patterson, K., Fryer, T. D., Hodges, J. R., Pengas, G., Williams, G. B., \& Nestor, P. J. (2011). Atrophy, hypometabolism and white matter abnormalities in semantic dementia tell a coherent story. Brain, 134(7), pp. 2025-2035.

Adlam, A.-L., Patterson, K., Rogers, T. T., Nestor, P. J., Salmond, C. H., Acosta-Cabronero, J., \& Hodges, J. R. (2006). Semantic dementia and fluent primary progressive aphasia: two sides of the same coin? Brain, 129(11), pp. 3066-3080.

Amblard, C., Lapalme, E., \& Lina, J.-M. (2004). Biomagnetic source detection by maximum entropy and graphical models. IEEE transactions on biomedical engineering, 51(3), pp. 427-442.

Ashburner, J. (2007). A fast diffeomorphic image registration algorithm. Neuroimage, 38(1), pp. 95113.

Ashburner, J., \& Friston, K. J. (2000). Voxel-based morphometry-the methods. Neuroimage, 11(6), pp. $805-821$.

Barulli, D., \& Stern, Y. (2013). Efficiency, capacity, compensation, maintenance, plasticity: emerging concepts in cognitive reserve. Trends in cognitive sciences, 17(10), pp. 502-509.

Benjamini, Y., \& Hochberg, Y. (1995). Controlling the false discovery rate: a practical and powerful approach to multiple testing. Journal of the royal statistical society. Series B (Methodological), pp. 289-300.

Besson, M., Magne, C., \& Regnault, P. (2004). Le traitement du langage. L'imagerie fonctionnelle électrique (EEG) et magnétique (MEG): Ses applications en sciences cognitives, pp. 185-216. 
Bi, Y., Wei, T., Wu, C., Han, Z., Jiang, T., \& Caramazza, A. (2011). The role of the left anterior temporal lobe in language processing revisited: evidence from an individual with ATL resection. cortex, 47(5), pp. 575-587.

Bier, N., Macoir, J., Joubert, S., Bottari, C., Chayer, C., Pigot, H., . . . Team, S. (2011). Cooking "Shrimp à la Créole": A pilot study of an ecological rehabilitation in semantic dementia. Neuropsychological rehabilitation, 21(4), pp. 455-483.

Binder, J. R., \& Desai, R. H. (2011). The neurobiology of semantic memory. Trends in cognitive sciences, 15(11), pp. 527-536.

Binder, J. R., Desai, R. H., Graves, W. W., \& Conant, L. L. (2009). Where is the semantic system? A critical review and meta-analysis of 120 functional neuroimaging studies. Cerebral cortex, 19(12), pp. 2767-2796.

Binney, R. J., \& Ralph, M. A. L. (2015). Using a combination of fMRI and anterior temporal lobe rTMS to measure intrinsic and induced activation changes across the semantic cognition network. Neuropsychologia, 76, pp. 170-181.

Brambati, S. M., Rankin, K., Narvid, J., Seeley, W., Dean, D., Rosen, H., . . Gorno-Tempini, M. L. (2009). Atrophy progression in semantic dementia with asymmetric temporal involvement: a tensorbased morphometry study. Neurobiology of aging, 30(1), pp. 103-111.

Catricalà, E., Della Rosa, P. A., Plebani, V., Perani, D., Garrard, P., \& Cappa, S. F. (2015). Semantic feature degradation and naming performance. Evidence from neurodegenerative disorders. Brain and language, 147 , pp. 58-65.

Chan, D., Anderson, V., Pijnenburg, Y., Whitwell, J., Barnes, J., Scahill, R., . . Rossor, M. N. (2009). The clinical profile of right temporal lobe atrophy. Brain, 132(5), pp. 1287-1298. 
Chen, Y., Chen, K., Ding, J., Zhang, Y., Yang, Q., Lv, Y., . . Han, Z. (2017). Brain network for the core deficits of semantic dementia: A neural network connectivity-behavior mapping study. Frontiers in human neuroscience, 11

Collins, J. A., Montal, V., Hochberg, D., Quimby, M., Mandelli, M. L., Makris, N., ... Dickerson, B. C. (2016). Focal temporal pole atrophy and network degeneration in semantic variant primary progressive aphasia. Brain, 140(2), pp. 457-471.

Cox, R. W. (1996). AFNI: software for analysis and visualization of functional magnetic resonance neuroimages. Computers and Biomedical research, 29(3), pp. 162-173.

Desgranges, B., Matuszewski, V., Piolino, P., Chételat, G., Mézenge, F., Landeau, B., . . Eustache, F. (2007). Anatomical and functional alterations in semantic dementia: a voxel-based MRI and PET study. Neurobiology of aging, 28(12), pp. 1904-1913.

Devlin, J. T., Russell, R. P., Davis, M. H., Price, C. J., Wilson, J., Moss, H. E., . . Tyler, L. K. (2000). Susceptibility-induced loss of signal: comparing PET and fMRI on a semantic task. Neuroimage, 11(6), pp. 589-600.

Diehl, J., Grimmer, T., Drzezga, A., Riemenschneider, M., Förstl, H., \& Kurz, A. (2004). Cerebral metabolic patterns at early stages of frontotemporal dementia and semantic dementia. A PET study. Neurobiology of aging, 25(8), pp. 1051-1056.

Diehl-Schmid, J., Onur, O. A., Kuhn, J., Gruppe, T., \& Drzezga, A. (2014). Imaging frontotemporal lobar degeneration. Current neurology and neuroscience reports, 14(10), p 489.

Federmeier, K. D., \& Kutas, M. (2005). Aging in context: age-related changes in context use during language comprehension. Psychophysiology, 42(2), pp. 133-141. 
Federmeier, K. D., Van Petten, C., Schwartz, T. J., \& Kutas, M. (2003). Sounds, words, sentences: agerelated changes across levels of language processing. Psychology and aging, 18(4), p 858.

Giaquinto, S., Ranghi, F., \& Butler, S. (2007). Stability of word comprehension with age: An electrophysiological study. Mechanisms of ageing and development, 128(11), pp. 628-636.

Gorno-Tempini, M. L., Hillis, A. E., Weintraub, S., Kertesz, A., Mendez, M., Cappa, S., . . Boeve, B. F. (2011). Classification of primary progressive aphasia and its variants. Neurology, 76(11), pp. 10061014.

Gorno-Tempini, M. L., Dronkers, N. F., Rankin, K. P., Ogar, J. M., Phengrasamy, L., Rosen, H. J., . . . Miller, B. L. (2004). Cognition and anatomy in three variants of primary progressive aphasia. Annals of neurology, 55(3), pp. 335-346.

Grabowski, T. J., Damasio, H., Tranel, D., Ponto, L. L. B., Hichwa, R. D., \& Damasio, A. R. (2001). A role for left temporal pole in the retrieval of words for unique entities. Human brain mapping, 13(4), pp. 199-212.

Gregory, S., Long, J. D., Klöppel, S., Razi, A., Scheller, E., Minkova, L., . . Leavitt, B. R. (2017). Operationalizing compensation over time in neurodegenerative disease. Brain, 140(4), pp. 1158-1165.

Grova, C., Makni, S., Flandin, G., Ciuciu, P., Gotman, J., \& Poline, J. (2006). Anatomically informed interpolation of fMRI data on the cortical surface. Neuroimage, 31(4), pp. 1475-1486.

Harbin, T. J., Marsh, G. R., \& Harvey, M. T. (1984). Differences in the late components of the eventrelated potential due to age and to semantic and non-semantic tasks. Electroencephalography and Clinical Neurophysiology/Evoked Potentials Section, 59(6), pp. 489-496. 
Hodges, J. R., \& Patterson, K. (2007). Semantic dementia: a unique clinicopathological syndrome. The Lancet Neurology, 6(11), pp. 1004-1014.

Hodges, J. R., Patterson, K., Oxbury, S., \& Funnell, E. (1992). Semantic dementia: progressive fluent aphasia with temporal lobe atrophy. Brain, 115(6), pp. 1783-1806.

Ikeda, M., Patterson, K., Graham, K. S., Ralph, M. L., \& Hodges, J. (2006). A horse of a different colour: do patients with semantic dementia recognise different versions of the same object as the same? Neuropsychologia, 44(4), pp. 566-575.

Jefferies, E., \& Lambon Ralph, M. A. (2006). Semantic impairment in stroke aphasia versus semantic dementia: a case-series comparison. Brain, 129(8), pp. 2132-2147.

Kumfor, F., Landin-Romero, R., Devenney, E., Hutchings, R., Grasso, R., Hodges, J. R., \& Piguet, O. (2016). On the right side? A longitudinal study of left-versus right-lateralized semantic dementia. Brain, 139(3), pp. 986-998.

Kutas, M., \& Federmeier, K. D. (2000). Electrophysiology reveals semantic memory use in language comprehension. Trends in cognitive sciences, 4(12), pp. 463-470.

Kutas, M., \& Federmeier, K. D. (2011). Thirty years and counting: finding meaning in the N400 component of the event-related brain potential (ERP). Annual review of psychology, 62, pp. 621-647.

Kutas, M., \& Hillyard, S. A. (1984). Brain potentials during reading reflect word expectancy and semantic association. Nature, 307(5947), pp. 161-163.

Kutas, M., \& Iragui, V. (1998). The N400 in a semantic categorization task across 6 decades. Electroencephalography and Clinical Neurophysiology/Evoked Potentials Section, 108(5), pp. 456471. 
Lau, E. F., Phillips, C., \& Poeppel, D. (2008). A cortical network for semantics:(de) constructing the N400. Nature reviews. Neuroscience, 9(12), p 920.

Luck, S. J. (2005). An introduction to the event-related potential technique MIT press. Cambridge, Ma, pp. 45-64.

Marinković, K. (2004). Spatiotemporal dynamics of word processing in the human cortex. The Neuroscientist, 10(2), pp. 142-152.

Marques, J. F., \& Charnallet, A. (2013). The role of feature sharedness in the organization of semantic knowledge: insights from semantic dementia. Neuropsychology, 27(2), p 266.

Mummery, C. J., Patterson, K., Price, C., Ashburner, J., Frackowiak, R., \& Hodges, J. R. (2000). A voxel-based morphometry study of semantic dementia: relationship between temporal lobe atrophy and semantic memory. Annals of neurology, 47(1), pp. 36-45.

Mummery, C. J., Patterson, K., Wise, R. J., Vandenbergh, R., Price, C., \& Hodges, J. (1999). Disrupted temporal lobe connections in semantic dementia. Brain, 122(1), pp. 61-73.

Murre, J. M., Graham, K. S., \& Hodges, J. R. (2001). Semantic dementia: relevance to connectionist models of long-term memory. Brain, 124(4), pp. 647-675.

Noonan, K. A., Jefferies, E., Visser, M., \& Ralph, M. A. L. (2013). Going beyond inferior prefrontal involvement in semantic control: evidence for the additional contribution of dorsal angular gyrus and posterior middle temporal cortex. Journal of Cognitive Neuroscience, 25(11), pp. 1824-1850.

Noppeney, U., \& Price, C. J. (2004). Retrieval of abstract semantics. Neuroimage, 22(1), pp. 164-170.

Papagno, C., \& Capitani, E. (2001). Slowly progressive aphasia: a four-year follow-up study. Neuropsychologia, 39(7), pp. 678-686. 
Patterson, K., Nestor, P. J., \& Rogers, T. T. (2007). Where do you know what you know? The representation of semantic knowledge in the human brain. Nature reviews. Neuroscience, 8(12), p 976.

Patterson, K., Ralph, M. A. L., Jefferies, E., Woollams, A., Jones, R., Hodges, J. R., \& Rogers, T. T. (2006). "Presemantic" cognition in semantic dementia: Six deficits in search of an explanation. Journal of Cognitive Neuroscience, 18(2), pp. 169-183.

Pobric, G., Jefferies, E., \& Ralph, M. A. L. (2007). Anterior temporal lobes mediate semantic representation: mimicking semantic dementia by using rTMS in normal participants. Proceedings of the National Academy of Sciences, 104(50), pp. 20137-20141.

Ralph, M. L. (2014). Neurocognitive insights on conceptual knowledge and its breakdown. Philosophical Transactions of the Royal Society of London B: Biological Sciences, 369(1634), p 20120392.

Ralph, M. L., Jefferies, E., Patterson, K., \& Rogers, T. T. (2017). The neural and computational bases of semantic cognition. Nat. Rev. Neurosci., 18, pp. 42-55.

Ralph, M. L., McClelland, J., Patterson, K., Galton, C., \& Hodges, J. (2001). No right to speak? The relationship between object naming and semantic impairment: Neuropsychological evidence and a computational model. Journal of Cognitive Neuroscience, 13(3), pp. 341-356.

Ralph, M. L., \& Patterson, K. (2008). Generalization and differentiation in semantic memory. Annals of the New York Academy of Sciences, 1124(1), pp. 61-76.

Rice, G. E., Lambon Ralph, M. A., \& Hoffman, P. (2015). The roles of left versus right anterior temporal lobes in conceptual knowledge: an ALE meta-analysis of 97 functional neuroimaging studies. Cerebral cortex, 25(11), pp. 4374-4391. 
Rogers, T. T., Hocking, J., Noppeney, U., Mechelli, A., Gorno-Tempini, M. L., Patterson, K., \& Price, C. J. (2006). Anterior temporal cortex and semantic memory: reconciling findings from neuropsychology and functional imaging. Cognitive, Affective, \& Behavioral Neuroscience, 6(3), pp. 201-213.

Rogers, T. T., Lambon Ralph, M. A., Garrard, P., Bozeat, S., McClelland, J. L., Hodges, J. R., \& Patterson, K. (2004). Structure and deterioration of semantic memory: a neuropsychological and computational investigation. Psychological review, 111(1), p 205.

Rogers, T. T., \& Patterson, K. (2007). Object categorization: reversals and explanations of the basiclevel advantage. Journal of Experimental Psychology: General, 136(3), p 451.

Rogers, T. T., Patterson, K., Jefferies, E., \& Ralph, M. A. L. (2015). Disorders of representation and control in semantic cognition: effects of familiarity, typicality, and specificity. Neuropsychologia, 76, pp. 220-239.

Schapiro, A. C., McClelland, J. L., Welbourne, S. R., Rogers, T. T., \& Ralph, M. A. L. (2013). Why bilateral damage is worse than unilateral damage to the brain. Journal of Cognitive Neuroscience, 25(12), pp. 2107-2123.

Scheller, E., Minkova, L., Leitner, M., \& Klöppel, S. (2014). Attempted and successful compensation in preclinical and early manifest neurodegeneration-a review of task fMRI studies. Frontiers in psychiatry, 5

Schmithorst, V. J., Dardzinski, B. J., \& Holland, S. K. (2001). Simultaneous correction of ghost and geometric distortion artifacts in EPI using a multiecho reference scan. IEEE transactions on medical imaging, 20(6), pp. 535-539. 
Seeley, W., Bauer, A., Miller, B., Gorno-Tempini, M., Kramer, J., Weiner, M., \& Rosen, H. (2005). The natural history of temporal variant frontotemporal dementia. Neurology, 64(8), pp. 1384-1390.

Smith, S. M., Matthews, P. M., \& Jezzard, P. (2001). Functional MRI: an introduction to methods: Oxford University Press.

Snowden, J. S., Goulding, P., \& Neary, D. (1989). Semantic dementia: a form of circumscribed cerebral atrophy. Behavioural Neurology

Snowden, J. S., Neary, D., \& Mann, D. (1996). Fronto-temporal lobar degeneration: fronto-temporal dementia, progressive aphasia, semantic dementia New York: Churchill Livingstone.

Taler, V., Klepousniotou, E., \& Phillips, N. A. (2009). Comprehension of lexical ambiguity in healthy aging, mild cognitive impairment, and mild Alzheimer's disease. Neuropsychologia, 47(5), pp. 13321343.

Tu, S., Leyton, C. E., Hodges, J. R., Piguet, O., \& Hornberger, M. (2016). Divergent longitudinal propagation of white matter degradation in logopenic and semantic variants of primary progressive aphasia. Journal of Alzheimer's Disease, 49(3), pp. 853-861.

Tyler, L. K., \& Moss, H. E. (2001). Concepts and categories: What is the evidence for neural specialisation? Behavioral and Brain Sciences, 24(3), pp. 495-496.

Tyler, L. K., Stamatakis, E. A., Bright, P., Acres, K., Abdallah, S., Rodd, J., \& Moss, H. (2004). Processing objects at different levels of specificity. Journal of Cognitive Neuroscience, 16(3), pp. 351362. 
Visser, M., Jefferies, E., \& Ralph, M. L. (2010). Semantic processing in the anterior temporal lobes: a meta-analysis of the functional neuroimaging literature. Journal of Cognitive Neuroscience, 22(6), pp. 1083-1094.

Visser, M., \& Ralph, M. L. (2011). Differential contributions of bilateral ventral anterior temporal lobe and left anterior superior temporal gyrus to semantic processes. Journal of Cognitive Neuroscience, 23(10), pp. 3121-3131.

Warrington, E. K. (1975). The selective impairment of semantic memory. The Quarterly journal of experimental psychology, 27(4), pp. 635-657.

Whitney, C., Kirk, M., O'sullivan, J., Lambon Ralph, M. A., \& Jefferies, E. (2010). The neural organization of semantic control: TMS evidence for a distributed network in left inferior frontal and posterior middle temporal gyrus. Cerebral cortex, 21(5), pp. 1066-1075.

Whitwell, J. L., \& Josephs, K. A. (2012). Neuroimaging in frontotemporal lobar degenerationpredicting molecular pathology. Nature Reviews Neurology, 8(3), pp. 131-142.

Wilson, S. M., Brambati, S. M., Henry, R. G., Handwerker, D. A., Agosta, F., Miller, B. L., . . GornoTempini, M. L. (2008). The neural basis of surface dyslexia in semantic dementia. Brain, 132(1), pp. 71-86. 